\title{
Computational Analysis of Ultra-Structural Images of Red Blood Cells
} Madhumita Das ${ }^{1,2}$, Bishwajit Das ${ }^{1,2}$, Avishek Das ${ }^{1,3}$, Ishita Chatterjee ${ }^{1,4}$ and Durjoy Majumder ${ }^{1,2 *}$

${ }^{1}$ Society for Systems Biology and Translational Research, Bangur Avenue, Kolkata, India

${ }^{2}$ Department of Physiology, West Bengal State University, Barasat, Kolkata, India

${ }^{3}$ Department of Information Technology, Adamas Institute of Technology, Barasat, Kolkata, India

${ }^{4}$ Department of Applied Psychology, University of Calcutta, Rajabazar Campus, Kolkata, India

\begin{abstract}
Light microscopic images are routinely used in clinical diagnosis. Further the confirmation of disease diagnosis is relied with the ultra-structural images. Scanning electron microscopy (SEM) with high resolution and high magnification is granted as one of the reliable process in this aspect. However, pathologists' criteria for a disease diagnosis for both the processes are on the basis of qualitative and empirical in nature. In a number of hematological diseases as well as in different pathological conditions like liver cirrhosis morphological alteration of red blood cell is reported. Recently some morphological alteration in red blood cells (RBC) by ultra-structural analysis has been reported in leukemic patients, though leukemia is a disease of white blood cells. It is expected that these features could be used as an event of cancer cachexia or can be used as the identifying marker for pre-cancer state. However, a systematic study so far is not done in terms of proper quantitative statement. Here an attempt has been made towards the development of an automated image analysis procedure for extracting quantitative information from the scanning electron microscopic (SEM) images. This computational approach may guide the clinicians to take a decision about the disease/pre-disease state with a quantitative outlook.
\end{abstract}

Keywords: Scanning electron microscopy; Image analysis; Hough transformation; Mathematical morphology; Leukemia

\section{Introduction}

It is well documented that in hematological malignancies abnormal cells of white blood cells are produced. In myeloid leukemia myeloid series of the hematopoetic system is affected in leukemia patients. Thus, different precursor cells of myeloid lineage like blast cells, promyelocytes, metamylocytes are observed in abundance in blood and bone marrow of leukemia patients. Similarly in lymphoid leukemia lymphoid blast cell and other precursor cells of lymphoid lineage are seen in abundance in blood and bone marrow in leukemia patient. Though there are different types of leukemia depending on lineage as mentioned but there is also mixed lineage leukemia where both the characteristic cells are seen.

Very recently attempts have been made to identify different characteristic signatures with red blood cells to identify the grading of malignancies. Very recently ultra-structural analysis of red blood cells proves this hypothesis in leukemia [1].

However, in earlier works people have attempted with an empirical approach and no quantitative analytical method has been developed still now. Here we made an attempt to establish a computational approach to extract information in a quantitative manner for those images.

Several computational works have been performed to extract information from images. For extracting image information, researchers have applied different image analysis methods for different fields such as, studying of paper coating layers [2], images of thin filament lengths have been analyzed using light microscopy images [3]. Quantitative measure of samples with various software tools can be made for this purpose [4]. Digital image analysis methods for cell image data in 2D, $3 \mathrm{D}$ and time sequences have recently been reported [5], which adopted a method to track stem cell over time, as stem cells have the capability to differentiate into specific cell types. In these works attempt has been made to make the automated procedure of image analysis that could be useful in automated diagnostic procedure.

CMYK (Cyan/ Magenta/ Yellow/ Key) method for quantitative image analysis has been reported with maximum gray component replacement, in which the lowest brightness level is subtracted from all channels [6]. Very recently algorithm has been developed to extract quantitative information about the light microscopic cellular images [7].

Contrarily ultra-structural images made through electron microscopy are grey-scale images. Grayscale images carry only intensity information. Each pixel is stored as a byte (value between 0 and 255). The computational analysis of heavily textured cellular transmission electron microscopy (TEM) images with typically low signal-to-noise ratio of neurons has been reported [8]. Image analysis of SEM images has been reported for testing a new optical metrology device to investigate the paper surface topography following pen and pencil strokes on different types of paper applied with various pressures [9]. An experimental study on maximum likelihood (ML) estimation of structure parameters from high-resolution transmission electron microscopy (HRTEM) images has also been reported [10].

Though we have used the same principles of image analysis, however, the use the statistical procedure to test the differences in different normal and disease samples may reveal the characteristic features of images through an automated procedure.

*Corresponding author: Durjoy Majumder, Department of Physiology, West Bengal State University, Berunanpukuria, Malikapur, Barasat, Kolkata 700 126, India, Tel: 033 2524 1979; E-mail: durjoy@rocketmail.com, durjoy@ssbtr.net

Received September 29, 2015; Accepted December 06, 2015; Published December 13, 2015

Citation: Das M, Das B, Das A, Chatterjee I, Majumder D (2015) Computational Analysis of Ultra-Structural Images of Red Blood Cells. J Oncol Transl Res 1: 104 10.4172/2476-2261.1000104

Copyright: (c) 2015 Das M, et al. This is an open-access article distributed under the terms of the Creative Commons Attribution License, which permits unrestricted use, distribution, and reproduction in any medium, provided the original author and source are credited. 


\section{Materials and Method}

\section{Image samples}

All scanning electron microscopic image samples utilized in this work are the same image samples of the earlier work [1]. All images are analyzed through Matlab 7.0 (The Mathworks, Inc.) [11].

\section{Mathematical morphology}

Mathematical morphology can be used as a tool to characterize size, convexity, connectivity, shape of objects that are useful in the representation and description of an image [12-14]. Operations are performed by combinations of a basic set of numerical manipulations between an image A and a small object $\mathrm{B}$, called structuring element. This is typically represented by a matrix of 0 s and $1 \mathrm{~s}$, which can be seen as a probe that scans the image and modifies it according to some specified rule, described in next subsection. The shape and size of $B$, typically much smaller than the image $A$, together with the specific rule, define the characteristics of the performed process.

The fundamental operations of morphological image processing are dilation and erosion. Both are defined in terms of the interaction of the original image A to be processed, and the structuring element $\mathrm{B}$.

Morphological dilation: Dilation is an operation that fills up objects in a binary image. Mathematically, dilation is defined as the set union of the objects A obtained after the translation of the original image for each coordinate pixel $b$ in the structuring element $B$ :

$$
A \oplus B=\bigcup_{b} \in_{B} T_{b}(\mathrm{~A})
$$

Binary dilation can be interpreted as the combination of two sets by using the vector additions of set elements, called the Minkowski Addition. This operation is expressed as:

$A \oplus B=\{r \mid r=a+b \forall a \in$ Aand $b \in B\}$

Morphological erosion: Erosion operator takes two pieces of data as inputs. The first is the image A which is to be eroded by the small structuring element B. It is defined in terms of the Minkowski subtraction as:

$$
A \theta B=\{r \mid(r+b) \in A \forall b \in B\}
$$

In terms of set intersection erosion can be defined as:

$$
A \theta B=\bigcap_{b} \in_{B} T_{-b}(A)
$$

Opening filter: The morphological opening of A by B, denoted as $\mathrm{A}^{\circ} \mathrm{B}$, is simply erosion of $\mathrm{A}$ by $\mathrm{B}$, followed by dilation of the result by $\mathrm{B}$ :

$$
A \circ B=(A \theta B) \oplus B
$$

Dilation tries to undo erosion operation. However, some details closely related with the shape and size of the structuring element will vanish. Furthermore, an object disappearing as consequence of erosion can not be recovered.

Closing Filter: Morphological closing is the dual of opening, is defined as dilation followed by an erosion operation using the same structuring element:

$$
A \bullet B=(A \oplus B) \theta B
$$

Opening and closing filters have been used as discriminators for filtering, segmentation, edge detection, differential counting, or numerical analysis of shapes.

\section{Hough transformation}

The Hough transform is a technique, which can be used to isolate features of a particular shape within an image. The classical Hough transform is most commonly used for the detection of regular curves such as lines, circles, ellipses, etc. A generalized Hough transform can be employed in applications where a simple analytic description of a feature(s) is not possible. The main advantage of the Hough transform technique is that it is tolerant of gaps in feature boundary descriptions and is relatively unaffected by image noise.

In the Hough transform, a curve is generated in polar $(r, \theta)$ space for each edge point in Cartesian space. The accumulator space is plotted rectangularly with $\theta$ as the abscissa and $r$ as the ordinate. Curves generated by collinear points in the gradient image intersect in peaks $(r, \theta)$ in the Hough transform space. These intersection points characterize the straight line segments of the original image.

\section{Image processing}

For images analysis, all steps have been carried out using Matlab. Samples are stained with color to improve the contrast of the images. Fundamental steps in image analysis are image acquisition, preprocessing (e.g., noise reduction), segmentation (threshold, watershed, etc.), feature extraction, analysis and evaluation. These steps can be further divided into several sub steps, which is discussed in next subsection.

Input SEM images are grayscale images. Hough transformation is used to marking the boundary of the cells present in the image. Gaussian filter has been used before generating the accumulation array of Hough transformation. Original SEM image (Figure 1A) is cropped to select a specific cell (Figure 1B). Then accumulation array is generated from Circular Hough Transform (Figure 1C). Finally the raw image with circles detected is generated (Figure 1D). Another cropped cell is used to identify both the inner and outer boundary of the cell (Figure 1E).

For counting the horns (protrusion from cell), from the original image (Figure 2A) a specific cell is selected and cropped (Figure 2B). Extended minima image (Figure 2C) of the cell is obtained by the following procedure:

The enhanced image is obtained by adding the top-hat image and subtracting the bottom-hat image from the original cell image. Then taking the complement of the enhanced image and extended minima of the complement image. This extended minima image consists of noise, which is reduced by filling the image (Figure 2D). From the opened area of the image necessary count of the horns are made (Figure 2E).

Each selected objects are marked to find the opened area bounded by the boundary marker. Morphological information thus is extracted and analyzed.

\section{Image analysis}

Hough transformation has been used to identify circular objects (cells). Boundary and center of radii of each object are marked. Data values like radius of each marked cells are extracted and their averages are calculated. There is horn like structures in disease cells of SEM images. Count of horns and their features has been collected (only from visible side, count may be doubled for the whole cell). Extracted values are shown in respective tables.

\section{Results}

There are 10 (ten) SEM images of RBC of leukemia patients 
Citation: Das M, Das B, Das A, Chatterjee I, Majumder D (2015) Computational Analysis of Ultra-Structural Images of Red Blood Cells. J Oncol Transl Res 1: 104. 10.4172/2476-2261.1000104

Page 3 of 4

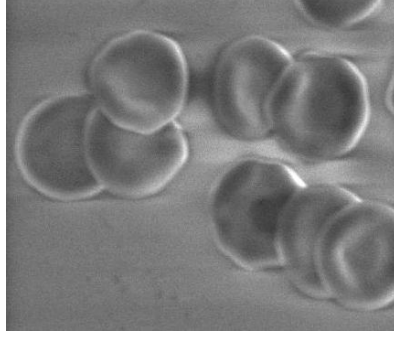

A

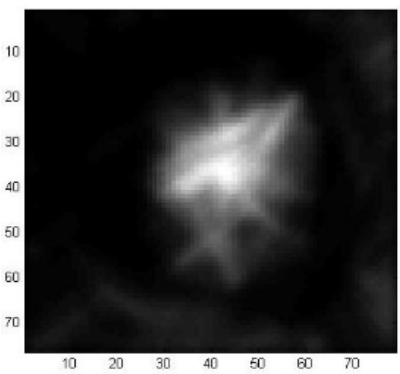

C

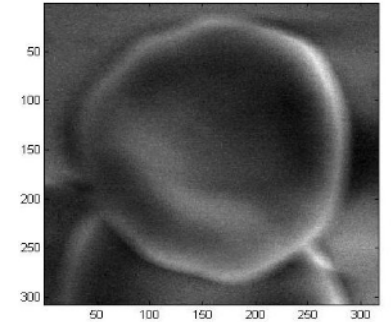

B

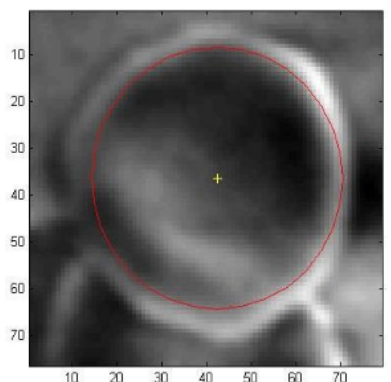

D

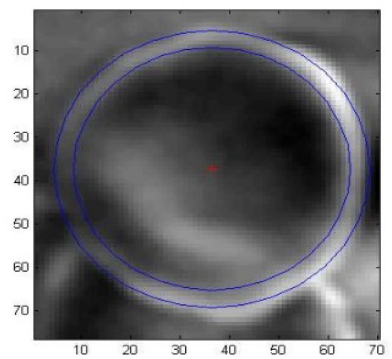

$\mathrm{E}$

Figure 1: Image processing for boundary marking and radius calculation. In A: Original SEM image; in B: cropped cell from the original image; in C: accumulation array from circular Hough transform; in D: Center of the cell and boundary is marked; and in E: center and both visible boundaries of the cell are marked.

used for horn count and 21 (twenty one) SEM images (12 of RBC of leukemia patients and 9 of RBC of normal volunteers, NV) used for radius calculation of cells (Table 1). Extracted data from each leukemic set is compared with data set of NV by Mann-Whitney U-test. Feature extracted from SEM images are also shown in table (Table 2). The data values are adjusted for 1000X magnification for all SEM images. The magnification factors of samples are noted in the respective tables.

\section{Discussion}

Leukemia is the malignancy of white blood cells and leukemia diagnosis followed by treatment procedure is mostly based on FAB classification. In our previous work we have established that in leukemia, RBCs are also affected [1]. This observation may be important in an understanding of the physiological process of cancer cachexia and grading or severity of leukemia and even pre-leukemic state for early management of disease, however for all these there is a necessity for development of an analytical methodology or algorithm. Here we have developed an algorithm based automated procedure for extracting the quantitative information from the scanning electron microscopy (gray scale) images. On analysis of SEM images, it was found that the

radius of the cells in ALL, AML, CLL and HPA samples are increased (Table 1) compare to RBC of normal volunteers, NV. These observations corroborate the previous empirical analysis (flattened structure of RBCs collected from leukemic patients) of the ultra-structural observation [1]. In the sample images of CML and MDS, most of the cells are clumped so values of individual cell diameter after Hough transformation may not produce distinct result, hence for those images we have slightly modify our algorithm. In these cases individual cells are cropped from the cell population, and then cell radius is calculated.

We have also developed algorithm for the analysis of horn structures present outside the cell structures - the parameters are number of horn count on the individual cell out of total area of individual cell. Here we assume that each cell is circular and area is calculated from radius [Cellular area is calculated by morphological operations]. Our analysis shows that there is a sharp distinction between RBCs of normal and leukemic samples (Table 1 and 2). Here the developed algorithm helps in the automated analysis of individual cells from an image consisting of a number of cells and quantitative estimation of structural abnormality

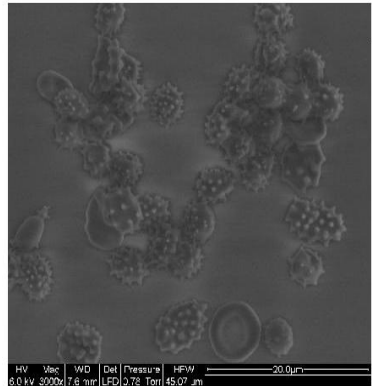

A

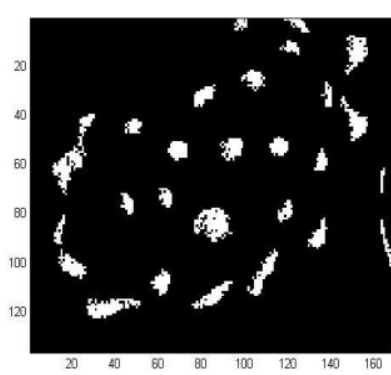

C

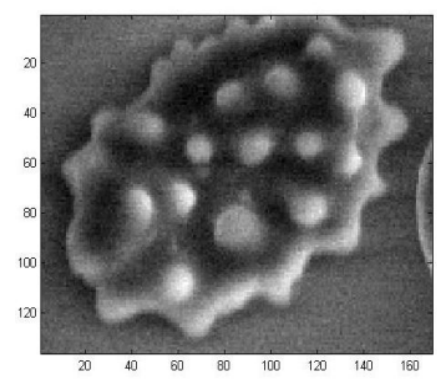

B

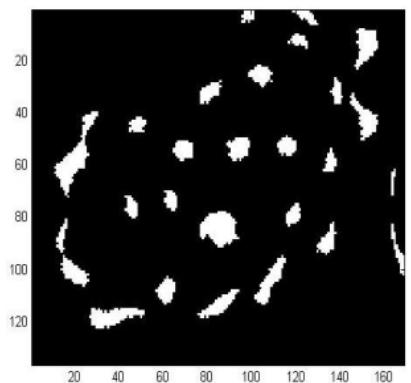

D

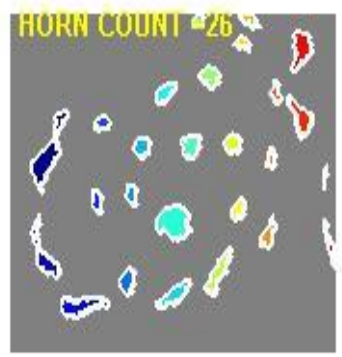

Figure 2: Horn counting and feature extraction of horn. In A: Original image for horn count; in B: Cropped cell from the original image; in C, Extended minima image of the cell; in D: Filled in image of the cell and in E: Horn count of the cell. 
Citation: Das M, Das B, Das A, Chatterjee I, Majumder D (2015) Computational Analysis of Ultra-Structural Images of Red Blood Cells. J Oncol TransI Res 1: 104. 10.4172/2476-2261.1000104

\begin{tabular}{|c|c|c|}
\hline Sample type & Analyzed cells Number & Mean \pm SD \\
\hline NV & 44 & $4.76 \pm 0.56$ \\
\hline ALL & 6 & $5.44 \pm 0.66(\mathrm{P}<0.05)$ \\
\hline AML & 14 & $5.24 \pm 0.61(\mathrm{P}<0.05)$ \\
\hline CLL & 20 & $4.91 \pm 0.64(\mathrm{P}<0.05)$ \\
\hline CML & 16 & $4.99 \pm 0.83(\mathrm{P}<0.05)$ \\
\hline HPA & 20 & $5.83 \pm 0.81(\mathrm{P}<0.01)$ \\
\hline MDS & 7 & $4.72 \pm 0.89(\mathrm{P}<0.05)$ \\
\hline
\end{tabular}

NV: Normal Volunteer; ALL: Acute Lymphoid Leukemia; AML: Acute Myeloid Leukemia; HPA: Hypoplastic Anemia; CLL: Chronic Lymphoid Leukemia; CML: Chronic Myeloid Leukemia; MDS: Myelodysplastic Syndrome

Table 1: Radius of cells after calibrated to 1000X.

\begin{tabular}{|c|c|c|c|}
\hline Sample type & Analyzed cells Number & Horn count & Horn count/cell \\
\hline NV & 76 & 47 & 0.61 \\
\hline ALL & $94(70)$ & 1117 & $11.88(15.95)$ \\
\hline CML & $41(9)$ & 156 & $3.80(17.33)$ \\
\hline MDS & $46(34)$ & 644 & $14.0(18.94)$ \\
\hline
\end{tabular}

Table 2: Horn count on RBC. Number in parentheses indicates only the morphologically abnormal cell.

(horn or thron counts) from an image consisting of a number of cells. Another advantage of this algorithm is that it can make a comparison between the results of two images that are captured with different magnifications.

\section{Acknowledgement}

M.D. and B.D. contributed equally to this work. All authors are thankful to Prof. S. Barat, Founder President of SSBTR for his constant encouragement for doing this work.

\section{References}

1. Majumder D, Banerjee D, Chandra S, Banerjee S, Chakrabarti A (2006) Red cell morphology in leukemia, hypoplastic anemia and myelodysplastic syndrome. Pathophysiology 13: 217-225.
2. Chinga G (2002) Structural studies of LWC paper coating layers using SEM and image analysis techniques, Ph.D. Thesis, Department of Chemical Engineering, Norwegian University of Science and Technology.

3. Ringkob TP, Swartz DR, Greaser ML (2004) Light microscopy and image analysis of thin filament lengths utilizing dual probes on beef, chicken and rabbit myofibrils. J Anim Sci, 82:1445-1453.

4. Vesela M, Zmeskal O, Vesely M, Nezadal M (2002) The use of Fractal Analysis for the Determination of Yeast Cell Diameter. HarFA - Harmonic and Fractal Image Analysis 21-22.

5. Pinidiyaarachchi A (2009) Digital Image Analysis of Cells - Applications in 2D, 3D and Time, PhD Thesis, Faculty of Science \& Technology, Uppsala University, Sweden, ISSN 1651-6214, ISBN 978-91-554-7398-3.

6. Pham N, Morrison A, Schwock J, Aviel-Ronen S, lakovlev V, et al. (2007) Quantitative image analysis of immunohistochemical stains using a CMYK color model. Diagnostic Pathology 2: 8.

7. Chattopadhyay M, Majumder D (2009) Computational approach for analysis of light microscopic images, Proc IEEE Int Conf Innov Tech (ICIT-09), Haryana, India.

8. Tasdizen T, Whitaker R, Marc R, Jones B (2005) Enhancement of cell boundaries in transmission electron microscopy images, Proc IEEE Int Conf Image Process (ICIP) 2005 II: 129-132.

9. Vernhes P, Passas R (2008) Optical Metrology and scanning electron microscopy of paper damage by writing, Microscopy and Analysis 22: S19-S22.

10. Van Aert S, Den Dekker AJ, Van Des Boss A, Van Dyke D, Chen JH (2005) Maximum likelihood estimation of structure parameters from high resolution electron microscopy images. Part-II: A practical example. Ultramicroscopy 2 : 107-125.

11. MATLAB user's guide, The Mathworks Inc.

12. Goutsias J, Vincent L, Bloomberg DS (2000) Mathematical morphology and its applications to image and signal processing. Kluwer Academic Publisher, New York, ISBN 0-792-37862-8.

13. Ledda A, Quintelier J, Samyn P, Baets PD, Philips W (2003) Quantitative image analysis with mathematical morphology, Proc ProRISC 2003, pp. 399-406.

14. Gonzalez RC, Woods RE, Eddins SL (2005) Digital Image Processing using MATLAB, Pearson Education, London. 